\title{
Coverage in WLAN with Minimum Number of Access Points
}

\author{
S. Kouhbor, J. Ugon, A. Rubinov, A. Kruger, M. Mammadov \\ School of Information Technology and Mathematical Sciences \\ University of Ballarat, Ballarat, Vic 3353 \\ skouhbor@students.ballarat.edu.au
}

\begin{abstract}
When designing wireless communication systems, it is very important to know the optimum numbers and locations for the access points (APs). The impact of incorrect placement of APs is significant. Placing too many access points increases the cost of deployment and interference, while placing too few access points can lead to coverage gaps. In this paper we describe a novel mathematical model developed to find the optimal number of APs and their locations in an environment that includes obstacles. To solve the problem, we use a new global optimization (AGOP) algorithm. The results obtained indicate that our model and software is able to solve optimal coverage problems for a design area with different number of users.
\end{abstract}

Keywords: WLAN, optimal number, optimization, coverage, access point, design area

\section{INTRODUCTION}

The primary goal of Wireless Local Area Network (WLAN) is to provide connectivity, or in other words coverage at all desired locations. Coverage is governed by the number and placement of APs.

The method used by network operators to find the placement of $\mathrm{AP}$ is based on the RF (radio frequency) site survey. This method is expensive and its results are not very reliable due to characteristics of the building and location of users that can change with time [1].

Another approach that is used by researchers [1]-[12] to find the optimal number and placement of APs is through the use of mathematical modeling and optimization techniques. Most of the authors [1]-[7] use discrete mathematical models to find the position of APs. In this case, the design area is divided into rectangles (grids). APs are only allowed to be placed in the centers of the rectangles. To obtain satisfactory results, the size of the grid must be sufficiently small. However, in this case, the dimension of the problem can be very high. For this reason some authors [8]-[12] prefer continuous mathematical models. In this approach, no restriction is placed on the position of APs.

The model investigated in the current paper is based on applying continuous optimization techniques to determine the minimum number of APs and their position for a design area that includes obstacles while coverage of the area is assured.

In general, small distances between transmitter and receiver and the type and number of obstructions (walls, furniture, door/or window) through which the radiated energy must pass, dominate indoor radio propagation. The presence of obstacles causes signal to reach a receiver through several paths with various strengths. Multi-path propagation causes the received signal, and hence the objective functions to be discontinuous. Solving these types of problems using existing optimization algorithms is very difficult.

We will use a nonsmooth optimization approach and the global optimization algorithm (AGOP) [13], [14]. It does not require gradient (or gradient like) information and can be applied to a wide range of nonsmooth functions.

The paper is organized as follows: Section II shows the model notation. Section III presents the optimization model. The solution method is described in Section IV. Results and the effect of AP parameters on coverage are discussed in Section V. The final section summarizes the paper and discusses future research.

\section{General Notations}

Throughout this paper the following notation is used:

$\begin{array}{ll}a_{j}, \quad j=1, \ldots, N & \text { Access points (AP) } \\ r_{i}, \quad i=1, \ldots, M & \text { Receivers/users } \\ d\left(a_{j}, r_{i}\right) & \text { Distance between AP and receiver } \\ g\left(a_{j}, r_{i}\right) & \text { Path loss from } i^{\text {th }} \text { user to access point } j \\ g_{\max } & \text { Maximum tolerable path loss } \\ n_{S P} & \text { Number of particular soft partitions } \\ l_{S P} & \text { Loss attributed to soft partition } \\ n_{H P} & \text { Number of particular hard partitions } \\ l_{H P} & \text { Loss attributed to hard partition } \\ P_{t} & \text { Transmit power } \\ R_{t h} & \text { Receive threshold } \\ a_{p} & \text { Position of AP } \\ t_{\mathrm{t}} & \text { Total processing time }\end{array}$

\section{OPtIMIZATION MODEL}

Some researchers [1]-[6], [8]-[10] have used path loss while others [11], [12] have used power at the receiver to develop their optimization problem. In this paper we follow the first approach to find the optimal placement of APs. Path loss model is the core of the signal coverage calculation for any environment [15]. It describes the loss of signal strength due to distance and obstacles between transmitter and receiver. Path loss models can be used to calculate the coverage area of 
$\mathrm{AP}$ and maximum distance between the two terminals in an ad hoc network.

\section{A. Model Description}

The objective function in this paper is based on path losses. The path loss for each receiver should satisfy the following condition:

$$
\min _{j} g\left(a_{j}, r_{i}\right) \leq g_{\max } \quad \forall i=1, \ldots, M
$$

These constraints state that path loss is evaluated against the maximum tolerable path loss $g_{\max }$. This ensures that the quality of coverage at each receiver location is above the given threshold. This given value, $g_{\max }$ can be calculated by subtracting receiver threshold from transmitter power.

$$
g_{\max }=P_{t}-R_{t h}
$$

The above inequality (1) can be expressed in the equality form as:

$$
\left(\min _{j} g\left(a_{j}, r_{i}\right)-g_{\max }\right)^{+}=0
$$

where $(\alpha)^{\dashv}=\max (\alpha, 0)$.

Define

$$
F_{1}\left(a_{1}, \ldots, a_{N}\right)=\sum_{i=1}^{M}\left(\min _{j} g\left(a_{j}, r_{i}\right)-g_{\max }\right)^{+}
$$

\section{B. Path Loss Model for Environments Including Obstacles}

Path loss function in a free space environment at a distance greater than the reference distance $d_{0}$ is given by [15]-[17]:

$$
g\left(a_{j}, r_{i}\right)[d B]=g\left(d_{0}\right)[d B]+10 \log \left(\frac{d\left(a_{j}, r_{i}\right)}{d_{0}}\right)^{2}
$$

where $g\left(d_{0}\right)$ is the path loss at reference distance.

In a typical building, signal may pass through walls, doors, windows, and furniture. The actual RF path between transmitter and receiver is affected by the type and number of obstructions existed in the path. Therefore, path loss including loss caused by partitions in (5) can be written as [15], [17]:

$$
\begin{aligned}
& g\left(a_{j}, r_{i}\right)[d B]=g\left(d_{0}\right)[d B]+10 \log \left(\frac{d\left(a_{j}, r_{i}\right)}{d_{0}}\right)^{2} \\
& +\sum n_{S P} l_{S P}+\sum n_{H P} l_{H P} .
\end{aligned}
$$

\section{SOLUTION OF THE PROBLEM}

The dependence of the received power on the position of users and AP is discontinuous. A move around a corner of the building or a wall (obstacle) can cause an entirely different pattern of reflected and transmitted rays. For most buildings, there will be many such points of discontinuity, distributed unpredictably. Solution of this type of problems using existing optimization algorithms is very difficult. For example, application of the discrete gradient (DG) algorithm [9]-[11] shows very good results, but it cannot be applied in the current setting.

Since objective function (4) is nonsmooth and nonconvex, traditional optimization algorithms (e.g. quasi-Newton method [8]) are not applicable either.

Direct Search methods [12] can be used for solving problems of type (4). However, for many such methods the number of variables and/or constraints, which can be efficiently handled, is restricted. It should also be noted that Direct Search methods are suitable for continuous functions.

We use a new global Optimization (AGOP) algorithm developed at the University of Ballarat to solve the problem. AGOP is designed for solving unconstrained continuous problems. It can be applied to a wide range of functions, requiring only function evaluations to work. In particular, it does not require gradient information and can be used to find minima of non-differentiable functions. Briefly, AGOP works as follows.

\section{A. Operation of $A G O P$ \\ Consider the problem:}

$$
\text { minimize } f(x): R^{n} \rightarrow R \text {, s.t } x \in B,
$$

where $B$ represents box constraints. AGOP must first be given an initial set of points $\Omega=\left\{x_{1}, \ldots, x_{q}\right\} \subset R^{n}$. Generally a suitable choice for an initial set is generated from the vertices of the box B. Suppose that $x_{*} \in \Omega$ has the smallest cost, that is, $f\left(x_{*}\right) \leq f(x)$ for all $x \in \Omega$. The set $\Omega$ and the values of $f$ at each of the points in $\Omega$ allow us to generate a dynamical system. This dynamical system determines a possible descent direction $v$ at the point $x_{*}$. An inexact line search along this direction provides a new point $\hat{x}_{q+1}$. A local search about $\hat{x}_{q+1}$ is then carried out. This is done using a direct search method called local variation. This is an efficient local optimization technique that does not explicitly use derivatives and can be applied to nonsmooth functions. Letting $x_{q+1}$ denote the optimal solution of this local search, the set $\Omega$ is augmented to include $x_{q+1}$. Starting with this updated $\Omega$, the whole process can be repeated. The process is terminated when $v$ is approximately 0 (or a prescribed bound on the number of iterations is reached). The solution returned is the current $x_{*}$, that is, the point in $\Omega$ with the smallest cost.

\section{B. Finding the Number and Location of $A P s$}

Initially we set the number of APs to 1 . Then the necessary number of APs is found through the following steps: 
1. Try to minimize the function $F_{1 ;}$

2. If the solution is 0 , then $N$ is the desired number;

3. Otherwise, $N$ is increased by 1: $N=N+1$;

4. Go to step 1.

\section{Method of Testing}

In this paper we report the results we obtained for a floor plan that was tested in [1]. This floor plan shown in Fig. 1 has dimension of $75 \mathrm{~m} \times 30 \mathrm{~m}$. There are two types of walls with loss of $6 \mathrm{~dB}$ and $3 \mathrm{~dB}$ shown with lines of different thickness. 223 users are distributed in the building. Specifications of one model of AP developed by Cisco (Aironet 1100 series) [18] is used to test the model and algorithm. This gives us the opportunity to examine the model not only for the two threshold values specified in [1], but also with wide range values of $P_{\mathrm{t}}$ and $R_{\mathrm{th}}$ to find the effect of AP parameters on the number of APs.

\section{Value of Path Loss at Reference Distance}

According to IEEE $802.11 \mathrm{~b}$ standard, carrier frequency $(f)$ is $2.4 \times 109 \mathrm{~Hz}$. We assumed that the reference distance $\left(d_{0}\right)$ is $1 \mathrm{~m}$. With these values in mind, path loss at reference distance, $g\left(d_{0}\right)$ is $40.04 \mathrm{~dB}$.

\section{E. Static AP and Mobile Users}

The goal of the solution to the optimization problem is to provide coverage for the design area. The location of users in the design area is set by the design specification, while the location and number of $\mathrm{APs}$ are to be found by the program. Once, the number and placement of APs are found, they are installed permanently. In order to make users mobile, coverage should be provided for all parts of the design area, that is, potential users should be placed in all parts of the building. Although users in the model and in the pictures seem static, they can move around because coverage is provided for the entire area.

\section{RESULTS}

In order to show the position of APs clearly, users are removed once the number and position of APs are found. Table I shows figure number, processing time and optimal number and placement of APs for some of the values of $P_{\mathrm{t}}$ and $R_{\text {th. }}$. Maximum tolerable path loss $\left(g_{\max }\right)$ is calculated using (2). When $P_{\mathrm{t}}=20 \mathrm{dBm}$ and $R_{\mathrm{th}}=-80 \mathrm{dBm}\left(g_{\max }=100 \mathrm{~dB}\right)$, our program found two APs that can cover all parts of the building (see Table 1,). This is the same as the result obtained in [1]. Fig. 2 shows the number and placement of APs. When $P_{\mathrm{t}}=20$ $\mathrm{dBm}$ and $R_{\mathrm{th}}=-60 \mathrm{dBm} \quad\left(g_{\max }=80 \mathrm{~dB}\right)$, a significant result was obtained: our program found 4 APs that can provide $100 \%$ coverage in contrast to 5 APs that was found in [1]. Fig. 3 shows placement of APs.

To make sure that total coverage is obtained, we connected users to their nearest APs and calculated their path loss using (6). If this path loss to any of the AP is less than $g_{\max }$, then we know that user is covered by that particular AP. To show this process, we provide two examples shown in Fig. 4. In this figure userl is connected to all APs. The calculated path loss for this user to AP3 and AP1 is more than $g_{\max }$ because of the type of walls that are separating this user from the two APs. The path loss to AP2 is $68.64 \mathrm{~dB}$ and to $\mathrm{AP} 4$ is $74.6 \mathrm{~dB}$. Hence, user 1 is covered by both AP2 and AP4. However, AP2 provides a stronger signal for this user than $\mathrm{AP} 4$ because of lower path loss. In the case where AP2 is out of order, user 1 can communicate with AP4. User2 provides the second example. This user is not covered by AP3 and AP4 because there are many walls between them. Path loss from this user to $\mathrm{AP} 2$ is $77.59 \mathrm{~dB}$ and to AP1 is $84.13 \mathrm{~dB}$. Therefore, we can conclude that user 2 can only communicate with AP2 because path loss is less than $g_{\max }$.

Further tests show that when $R_{\mathrm{th}}$ decreases to lower values such as $-95 \mathrm{dBm}$, while $P_{\mathrm{t}}$ is kept at $20 \mathrm{dBm}$, one AP can cover the area. If $P_{\mathrm{t}}$ decreases, while $R_{\mathrm{th}}$ remains at $-60 \mathrm{dBm}$, more $\mathrm{APs}$ are required. This is due to coverage distance that is decreasing. For example, Fig. 5 shows that when $P_{\mathrm{t}}=10 \mathrm{dBm}$ and $R_{\mathrm{th}}=-60 \mathrm{dBm}$, coverage of the area is assured by $8 \mathrm{APs}$. If $R_{\mathrm{th}}$ is reduced further to $-55 \mathrm{dBm}, 14 \mathrm{APs}$ are required to cover the entire area. This test shows that the capacity for users can be increased by decreasing the $P_{\mathrm{t}}$ and increasing $R_{\mathrm{th}}$.

\section{B. Effect of Number of Users on the Number of APS}

In order to further decrease the cost of deployment, we decided to provide coverage for the public areas instead of the entire area. This is applicable in places where coverage of places such as offices that are equipped with $\mathrm{PCs}$ is not necessary. To conduct this test, we removed users that are located in small rooms (offices). Fig. 6 shows that when $P_{\mathrm{t}}=$ $10 \mathrm{dBm}$, and $R_{\mathrm{th}}=-60 \mathrm{dBm}, 6 \mathrm{APs}$ are sufficient to cover users in contrast to $8 \mathrm{APs}$ that is necessary to cover entire area. With $P_{\mathrm{t}}=20 \mathrm{dBm}$ and $R_{\mathrm{th}}=-60 \mathrm{dBm}$, users are covered with 3 APs instead of 4 APs in the first case.

Number of users was further decreased to 158 by removing the ones that are in hallways. This didn't affect the number of APs when $P_{\mathrm{t}}$ is high. This is due to distribution of users and large coverage distance of APs. Effect could be observed if users are distributed in clustering format. However, Fig. 7 shows that when $P_{\mathrm{t}}$ is reduced to $10 \mathrm{dBm}$, and $R_{\mathrm{th}}$ is kept at -60 $\mathrm{dBm}$, number of APs is further reduced to 5 . This is due to coverage distance that is decreasing.

\section{Optimal Number of APs}

In order to determine if the solution obtained by our software is optimal, we conducted an exhaustive enumeration tests. The procedure we adopted is; first we removed one of the APs in order to distinguish users who were covered by removed AP. Then the position of remaining APs is changed for so many times to find if they can cover all users. We show our procedure in an example where $P_{\mathrm{t}}=10 \mathrm{dBm}$ and $R_{\mathrm{th}}=-94$ $\mathrm{dBm}$. Fig. 8 shows the position of APs. When coverage distance of these two APs was calculated, it appeared that one AP should be sufficient. By removing AP1, we found users who were covered by this AP. This is shown in Fig. 9. Then, AP2 was moved to many positions aiming to find the best place where it could cover all users. After many trials, it was 
concluded that one AP is not sufficient. Fig. 10 shows two users on the right that could not be covered by AP2. It was noted that if the loss associated to the wall shown in Fig. 10 is changed from $6 \mathrm{~dB}$ to $3 \mathrm{~dB}$, then building (all users) could be covered by one AP when $P_{\mathrm{t}}=10 \mathrm{dBm}$ and $R_{\mathrm{th}}=-94 \mathrm{dBm}$.

\section{Processing Time}

Condition of experiments. Tests were conducted on a PC that is equipped with Pentium 4 processor, CPU of $3 \mathrm{GHz}$, and $496 \mathrm{MB}$ of RAM. Codes are written in $\mathrm{C}++$.

As it is shown in table $\mathrm{I}$, total processing time for finding 8 APs and their location when $P_{\mathrm{t}}=10 \mathrm{dBm}$ and $R_{\mathrm{th}}=-60 \mathrm{dBm}$ is 644.18 seconds. In the case where $P_{\mathrm{t}}=20 \mathrm{dBm}$ and $R_{\mathrm{th}}=-95$ $\mathrm{dBm}$, processing time to find 1 APs and its location is 2.46 seconds. As can be seen in table II, the processing time depends on the number of users and obstacles in the area

\section{CONCLUSION}

This paper investigates a novel approach to coverage problem in WLAN based on minimizing a nonnegative objective function that is 0 only when the coverage of the entire area is assured. We used a new global optimization algorithm to solve the mathematical model. The model was tested using different values of $P_{\mathrm{t}}$ and $R_{\mathrm{th}}$. The results obtained indicate that the model described above can be used for finding the optimal number and placement of APs while covering all parts of design area. It is observed that the dimension of the building, number of users and their locations and the value of $P_{\mathrm{t}}$ and $R_{\mathrm{th}}$ has an effect on the number and placement of APs needed to cover an area. As the power of AP decreases, the coverage distance decreases as well. Hence, more APs are required to cover area/users as it was expected. This feature can be used to increase capacity for the users who are performing critical activities.

Further research will involve in enhancing the security of the network by controlling the amount of radiation of APs beyond the design area. When APs are kept away from exterior walls, intruders will have less chance in accessing the network. We will also investigate the effect of shadow fading on the number of $\mathrm{APs}$, limiting number of users per $\mathrm{AP}$ in order to increase capacity for each user, and limiting the overlapping of APs.

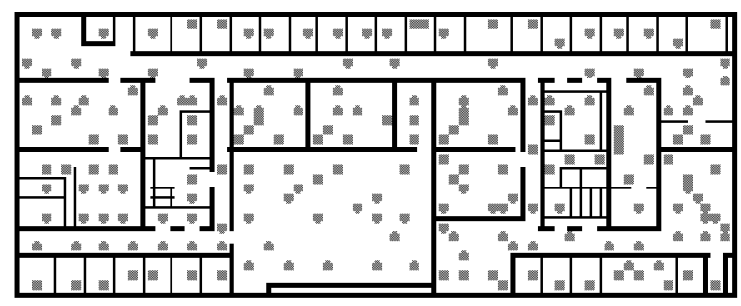

Fig. 2. Layout of the design area and position of users
TABLE I. NUMBER AND PLACEMENT OF APs FOR ENTIRE DESIGN AREA

\begin{tabular}{|c|c|c|c|c|}
\hline$P_{\mathrm{t}}(\mathrm{dBm})$ & $R_{\mathrm{th}}(\mathrm{dBm})$ & $a_{p}$ & $t_{t}(\mathrm{Sec})$ & Fig. \\
\hline 20 & -95 & {$[42.05,9.46]$} & 2.46 & \\
\hline 20 & -80 & {$[25.6,12.89][72.97,7.44]$} & 12.84 & 2 \\
\hline 20 & -60 & $\begin{array}{l}{[19.28,6.61][54.86,22.02]} \\
{[70.4,9.42][20.01,24.12]}\end{array}$ & 113 & 3 \\
\hline 20 & -55 & $\begin{array}{l}{[68.1,7.08][60.65,23.35]} \\
{[9.73,22.7][34.97,25.1][40.36,9.9]} \\
{[10.85,4.54]}\end{array}$ & 300.23 & - \\
\hline 10 & -60 & $\begin{array}{l}{[33.5,5.6][41.7,23.03]} \\
{[21.72,26.12][63.76,22.94]} \\
{[12.87,4.76][68.67,4.92]} \\
{[54.12,13.66][13.91,17.68]}\end{array}$ & 644.18 & 5 \\
\hline
\end{tabular}

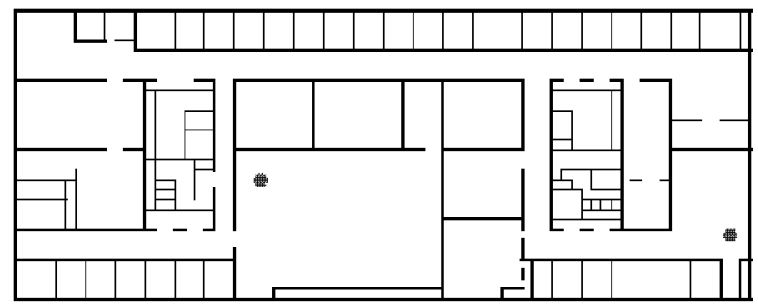

Fig. 2. Optimal number of APs when $P_{\mathrm{t}}=20$ and $R_{\mathrm{th}}=-80 \mathrm{dBm}$

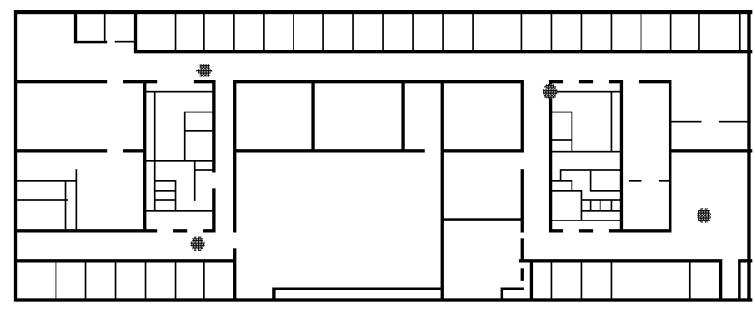

Fig. 3. Optimal number of APs when $P_{\mathrm{t}}=20$ and $R_{\mathrm{th}}=-60 \mathrm{dBm}$

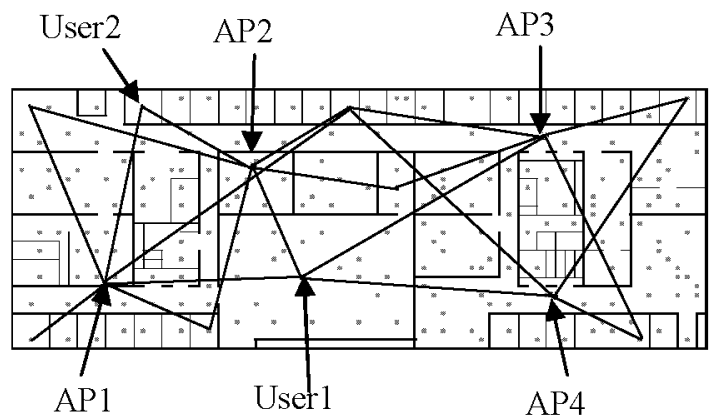

Fig. 4. Connection of users to their nearest APs

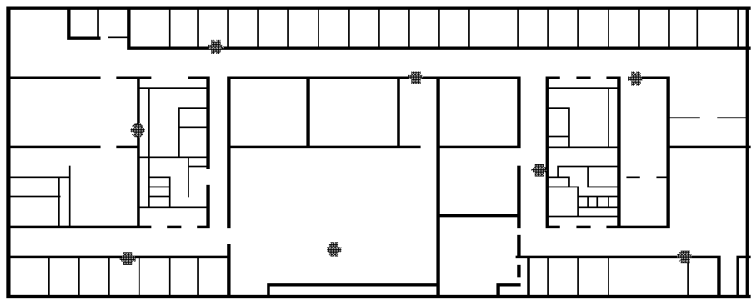

Fig. 5. Optimal number of APs when $P_{\mathrm{t}}=10$ and $R_{\mathrm{th}}=-60 \mathrm{dBm}$ 


\section{REFERENCES}

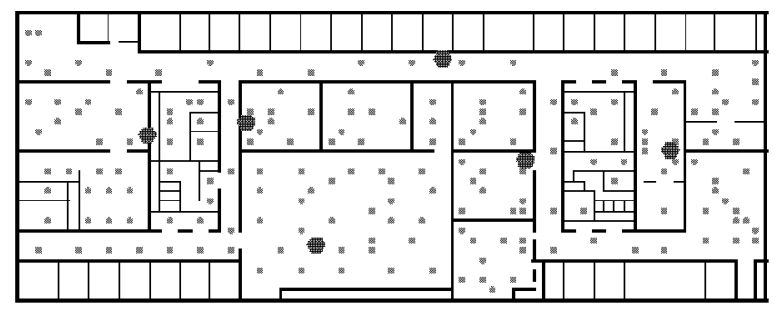

Fig. 6. Coverage of 183 users when $P_{\mathrm{t}}=10$ and $R_{\mathrm{th}}=-60 \mathrm{dBm}$

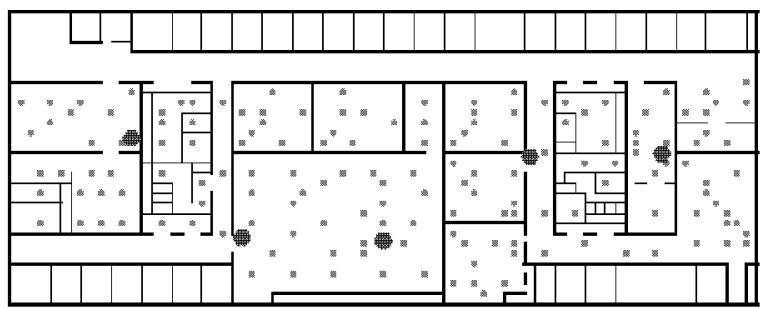

Fig. 7. Coverage of 158 users when $P_{\mathrm{t}}=10$ and $R_{\mathrm{th}}=-60 \mathrm{dBm}$

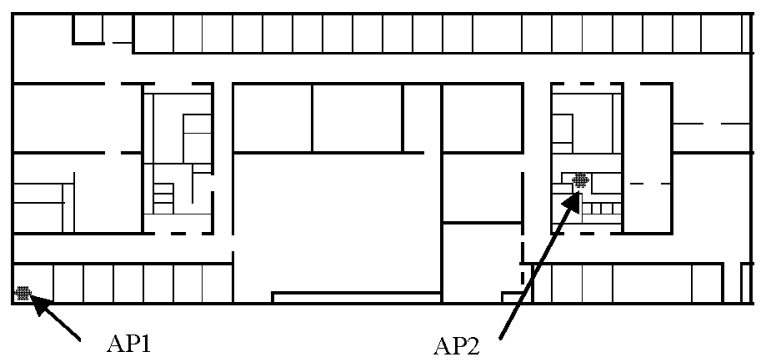

Fig. 8. Optimal number of APs when $P_{\mathrm{t}}=10$ and $R_{\mathrm{th}}=-94 \mathrm{dBm}$

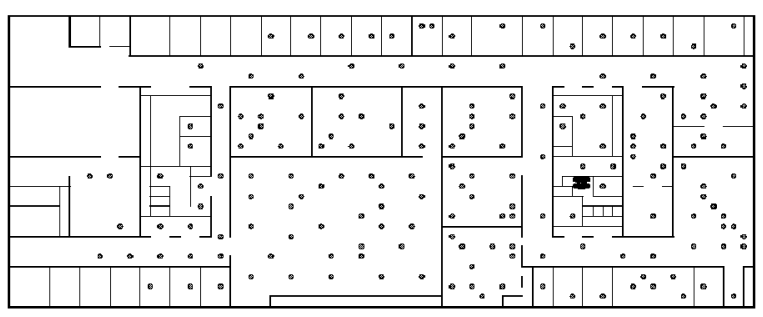

Fig. 9. Removed users who were covered by AP1

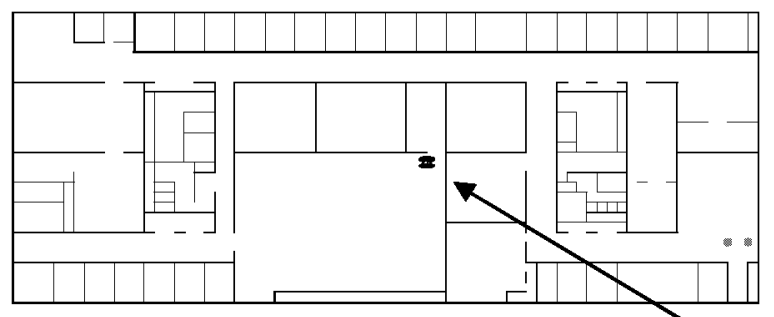

Fig. 10. Users that cannot be covered by one AP Wall with loss of $6 \mathrm{~dB}$
[1] M. D. Adickes, R. E. Billo, B. A. Norman, S. Banerjee, B. O. Nnaji, and J. Rajgopal, "Optimization of Indoor Wireless Communication Network Layouts," IIE (Institute of Industrial Engineers) Transactions, vol. 34, pp. $823-836,2002$.

[2] M. Kamenetsky and M. Unbehaun, "Coverage Planning for Outdoor Wireless LAN Systems," in Proc. IEEE International Zurich Seminar on Broadband Communications, Sweden, 2002, pp.49-1 - 49-6.

[3] K.-S. Tang, K.-F. Man, and S. Kwong, "Wireless Communication Network Design in IC Factory," IEEE Transaction on Industrial Electronics, vol. 48, pp. 452 - 458, 2001.

[4] M. Unbehaun and M. Kamenetsky, "On the Deployment of Picocellular Wireless Infrastructure," in IEEE Wireless Communication Magazine, 2003, vol. 10, pp. $70-80$.

[5] B.-S. Park, J.-G. Yook, and H.-K. Park, "The Determination of Base Station Placement and Transmit Power in an Inhomogeneous Traffic Distribution for Radio Network Planning," in Proc. IEEE 56th Vehicular Technology Conference, 2002, vol. 4, pp. 2051-2055.

[6] H. R. Anderson and J. P. McGechan, "Optimizing Microcell Base Station Locations Using Simulated Annealing Techniques," in Proc. 44th Vehicular Technology Conference, 1994, pp. 858 - 862.

[7] Y. Lee, K. Kim, and Y. Choi, "Optimization of AP Placement and Channel Assignment in Wireless LANs," in Proc. 27th Annual IEEE Conference on Local Computer Networks (LCN'02), 2002.

[8] H. D. Sherali, C. M. Pendyala, and T. S. Rappaport, "Optimal Location of Transmitters for Micro-Cellular Radio Communication System Design," IEEE Journal on Selected Areas in Communications, vol. 14, pp. 662 $673,1996$.

[9] S. Kouhbor, J. Ugon, A. Kruger, A. M. Rubinov, and P. Branch, "A New Algorithm for the Placement of WLAN Access Points Based on Nonsmooth Optimization Technique," in Proc. $7^{\text {th }}$ Intemational Conference on Advanced Communication Technology (IEEE/ICACT2005), 2005, Vol. 1, pp.352-357.

[10] S. Kouhbor, J. Ugon, A. Kruger, A. M. Rubinov, "Optimal Placement of Access Point in WLAN Based on a New Algorithm," in Proc. $4^{\text {th }}$ International Conference on Mobile Business (IEEE/ICMB 2005), 2005, pp. 592-598.

[11] S. Kouhbor, J. Ugon, A. Kruger, A. Rubinov, and P. Branch, "Optimization in Wireless Local Area Network," presented at 6th International Conference on Optimization: Techniques and Applications (ICOT A6), Ballarat, Australia, 2004.

[12] S. Fortune, D. Gay, B. Kernigban, O. Landron, R. Valenzuela, and M Wright, "Wise Design of Indoor Wireless System: Practical Computation and Optimization, "IEEE Computational Science and Engineering, 1995, pp. $58-68$.

[13] M. Mammadov, "A New Global Optimization Algorithm Based on a Dynamical Systems Approach," presented at International Conference on Optimization: Techniques and Applications - ICOTA6, 2004

[14] M. Mammadov, A. Rubinov, and J. Yearwood, "Dynamical Systems Described by Relational Elasticities with Applications", In Continuous Optimization: Current Trends and Applications, V.Jeyakumar and A.Rubinov (eds), Springer, 2005

[15] K. Pahlavan and P. Krishnamurthy, Principles of Wireless Networks: A Unified Approach: Prentice - Hall, Ine., 2002.

[16] T. S. Rappaport, Wireless Communications: Principles and Practice, Second ed: Prentice - Hall, Inc, 2002.

[17] M. A. Panjwani, A. L. Abbott, and T. S. Rappaport, "Interactive Computation of Coverage Regions for Wireless Communication in Multifloored Indoor Environments," IEEE Joumal on Selected Areas in Communications, vol. 14, pp. 420 - 430, 1996.

[18] Cisco, "Cisco Aironet 1100 Series Access Point data sheet," Cisco Systems, 2004 